\title{
Automation-Assisted Complementary Techniques for Multi-Spectroscopy TEM and STEM Spatially-Resolved Microanalysis
}

\author{
A. Aitouchen, S. T. Kim and J. K. Weiss \\ Emispec, 2050 S. Cottonwood Dr., Tempe, AZ 85282
}

Spectrum imaging and energy-filtered imaging are now established and indispensable tools in materials science characterization. TEM mode EELS images (EFTEM) and STEM mode EELS or EDX spectrum images are routinely used individually, but are rarely combined. By using integrated software that supports all of the microscope modes and spectroscopy types, it is possible to take advantage of their complementary nature. EFTEM is good for large-area semi-quantitative elemental maps [1] while STEM microanalysis is ideal for accurate quantitative analysis from very small areas [2]. EELS and EDX also offer complementary information because of the wide variation in sensitivity to different elements for each type of spectroscopy. The ability to automatically acquire correlated TEM and STEM images opens the possibility of using EFTEM elemental maps for locating specific areas from which quantitative compositional information can be acquired using STEM microanalysis. The key advantage to such an approach over full spectrum imaging is that the total time required for the analysis is at least one order of magnitude lower than that required to acquire the full spectrum image. The quality of the quantitative analysis can also be significantly higher, especially for small particles, since the dwell time for the point analysis would usually much higher than the pixel time for a spectrum image.

Fig. 1 illustrates the use of EFTEM imaging combined with STEM EDX analysis using an $\mathrm{Al}_{2} \mathrm{O}_{3} /$ TiC composite sample. It is not obvious from the bright field TEM image which grains are of a particular composition, whereas the elemental maps show distinct compositional contrast, including several small Ti-rich grains embedded in an $\mathrm{Al}_{2} \mathrm{O}_{3}$ grain. After the bright field STEM image was accurately aligned with the bright field TEM image, the coordinates of the positions indicated in Fig. $1 \mathrm{~b}$ and $1 \mathrm{c}$ were then used for positioning the probe for STEM microanalysis. Fig $1 \mathrm{~d}$ shows the spectra acquired in STEM mode from the indicated positions.

Using the Emispec Cynapse platform [3], we are developing automation for various steps of this procedure. First, the reference position(s) for STEM microanalysis are automatically evaluated by using intensity thresholding in any one of the EFTEM elemental maps. Then the alignment of the TEM and STEM images is automated by using the premeasured shift, rotation, and scaling between TEM and STEM mode combined with fine adjustments measured using standard image registration techniques. Finally, the spectrum acquisition is done with either a fixed or a rastered probe in each selected area, or a full spectrum image is acquired from each area.

There is a large class of samples for which this approach is particularly applicable. This would include small widely distributed particles, such as precipitates or supported metal catalysts, and systems with distinct areas of different composition, such as semiconductor devices or ceramic composites. Additionally, acquiring both EDX and EELS can help where there is significant peak overlap for some elements and where thickness variations that affect EDX quantification can be measured with EELS.

\section{References}

[1] J. Bentley, Microscopy \& Microanalysis 4 (1998) 158.

[2] A. Aitouchen, Microscopy \& Microanalysis 7 (2001) 1168.

[3] J. K. Weiss and W. J. de Ruijter, Microscopy \& Microanalysis (this issue) (2003). 

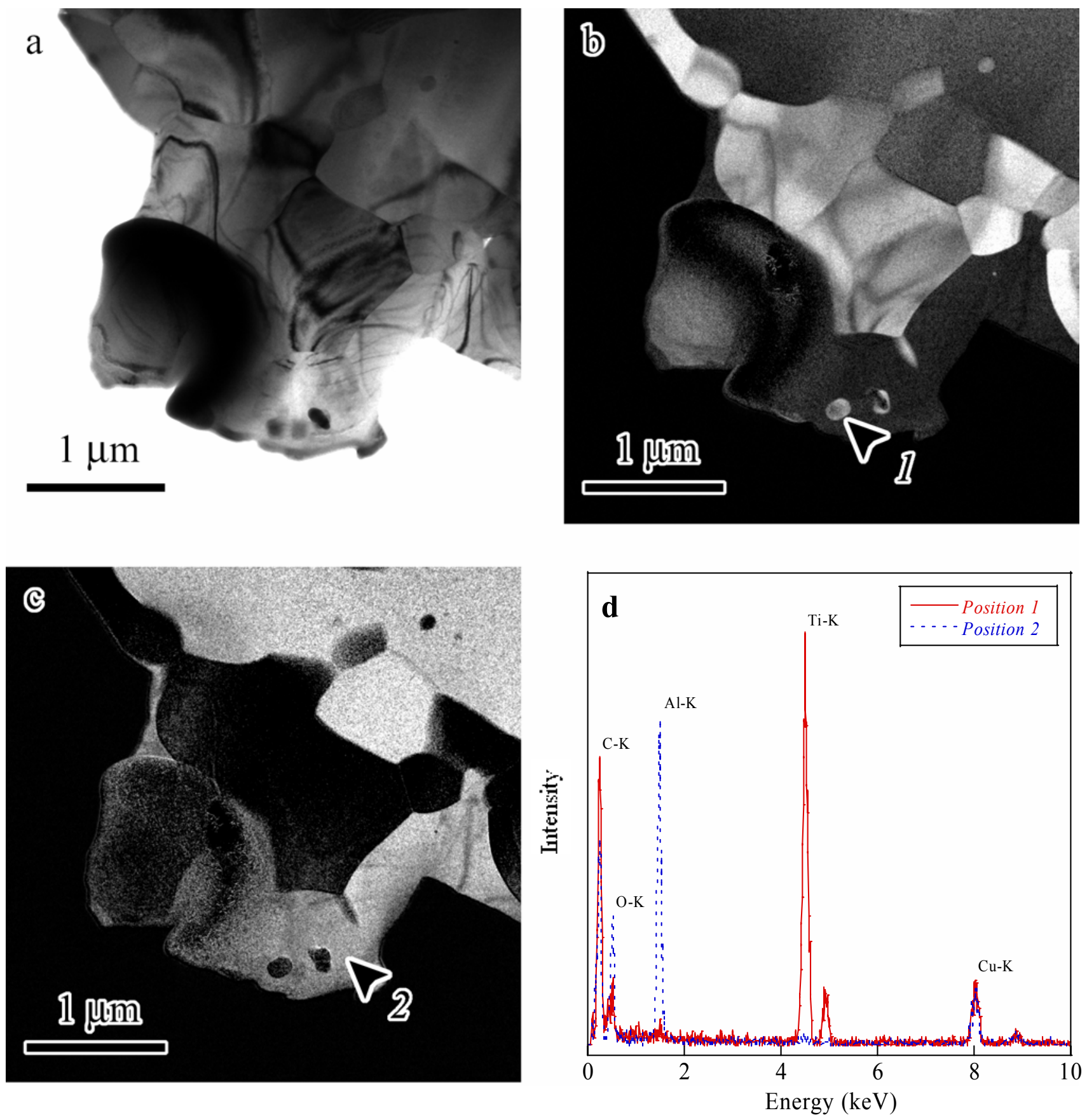

FIG. 1. Zero-loss filtered image (a) of $\mathrm{Al}_{2} \mathrm{O}_{3} / \mathrm{TiC}$ composite, and EFTEM elemental maps for $\mathrm{Ti}$ (b), and $\mathrm{O}(\mathrm{c})$. EDX spectra (d) were acquired in STEM mode from the positions 1 and 2, indicated in $1 \mathrm{~b}$ and $1 \mathrm{c}$, respectively. 